\section{Natalizumab-induced PML: can the beast be tamed?}

\author{
Reinhard Hohlfeld ${ }^{1,2}$
}

Professor Ralf Gold and his team ${ }^{1}$ report on a cohort of 15 patients with natalizumab-associated progressive multifocal leucoencephalopathy (PML) who were treated at the university hospital of Bochum, Germany. This is the largest PML series from a single institution reported so far. It comprises almost 5\% of the total PML cases presently known worldwide. The most remarkable observation is that none of the patients died during 21.5 months of median follow-up. By contrast, the overall lethality of natalizumab-associated PML is currently about 20\%. What can we learn from these findings?

1. The earlier PML is diagnosed, the better the prognosis. In the Bochum series, the mean interval from the first clinical and/or MRI evidence to the definite diagnosis by John Cunningham virus (JCV)-DNA detection in the cerebrospinal fluid (CSF) was 30 days. In two patients, suspicious lesions were detected on routine MRI without accompanying clinical symptoms.

2. Treatment should begin as soon as the diagnosis is made. As an initial step, all patients received a course of plasmapheresis or immunoabsorption to remove circulating natalizumab. ${ }^{2}$ In addition, they were treated with mefloquine and mirtazapine, both of which have anti-JCV effects in vitro, although evidence for in vivo efficacy is lacking.

3. Seizures should be controlled rigorously by antiepileptic treatment. Notably, $50 \%$ of the Bochum patients developed focal or generalised seizures during the

\footnotetext{
${ }^{1}$ Institute of Clinical Neuroimmunology, Ludwig Maximilians University, Munich, Germany; ${ }^{2}$ Munich Cluster of Systems Neurology (SyNergy), Munich, Germany

Correspondence to Professor Reinhard Hohlfeld, Institute of Clinical Neuroimmunology, Klinikum der LMU München, Campus Grosshadern, Marchioninistr. 15, München D-81377, Germany; reinhard.hohlfeld@med.uni-muenchen.de
}

acute PML phase; one patient even presented with generalised seizures as the first sign of PML. Despite intense treatment, epilepsy could be only incompletely controlled in four of the eight patients who developed seizures.

4. Early recognition and treatment of immune reconstitution inflammatory syndrome (IRIS) is crucial for survival. All patients developed some form of IRIS within a mean interval of 32 days after plasmapheresis/immunoadsorption.

Clinical deterioration was preceded or accompanied by early or late enhancement of PML MRI lesions. Only one patient had a clinically indolent course of IRIS. Following current recommendations, IRIS was treated with pulsed high-dose steroids; two patients received mannitol for brain oedema.

5. It may be difficult to distinguish between ongoing PML, PML-IRIS and multiple sclerosis (MS) exacerbation by MRI. In such cases, a brain biopsy may be helpful in therapeutic decisions ${ }^{3}$ (this was not necessary in the Bochum series).

6. Detection of JCV-DNA by quantitative PCR (qPCR) remains the gold standard for the diagnosis of natalizumabassociated PML. Rarely, qPCR remains negative despite typical clinical and MRI signs of PML, as was the case in one of the patients. ${ }^{1}$ It should be noted that the CSF viral load may transiently increase during the IRIS phase (11/15 patients).

7. After removal of natalizumab, MS activity eventually recurs in the majority of PML survivors. In the Bochum series, two-thirds of the patients showed MRI and/or clinical MS activity after a mean interval of 11 months from the PML diagnosis. The majority of these patients were treated with glatiramer acetate because they had previously failed to respond to interferon- $\beta$; one patient was treated with interferon- $\beta$ and two with fingolimod. One-third of the patients remained stable without immunotherapy.
In conclusion, it is highly encouraging that, with proper treatment and special attention to IRIS, early and intermediateterm lethality of PML may be reduced to zero. Still, PML is a severe and disabling condition: $60 \%$ of the post-PML patients in the Bochum series were moderately to severely disabled, and the majority became dependent on external support. There is reason for optimism that, in the future, the incidence of natalizumabinduced PML can be reduced by stratifying patients, for example, according to anti-JCV antibody status. ${ }^{4}$ Another big challenge is to investigate treatment strategies for controlling MS in the hopefully (presumably) increasing number of postPML patients who survive and recover despite possible persistence of the virus. ${ }^{5}$

Competing interests $\mathrm{RH}$ has received personal compensation for being on the advisory board of and/ or as consultancy fees from Teva, Sanofi, Genzyme, Bayer/Schering, Merck-Serono, Biogen-Idec, Novartis, Behring CSL, Morphosys; research grant support from Teva, Bayer/Schering, Serono, Biogen-Idec, Novartis.

Provenance and peer review Commissioned internally peer reviewed.

To cite Hohlfeld R. J Neurol Neurosurg Psychiatry 2013;84:1065

Received 8 February 2013

Accepted 9 February 2013

Published Online First 9 March 2013

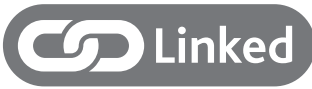

http://dx.doi.org/10.1136/jnnp-2013-304897

J Neurol Neurosurg Psychiatry 2013;84:1065. doi:10.1136/jnnp-2013-305077

\section{REFERENCES}

1 Dahlhaus S, Hoepner R, Chan A, et al. Disease course and outcome of 15 monocentrically treated natalizumab-associated progressive multifocal leukoencepahlopathy patients. J Neurol Neurosurg Psychiatry 2013;84:1068-73.

2 Kappos L, Bates D, Edan G, et al. Natalizumab treatment for multiple sclerosis: updated recommendations for patient selection and monitoring Lancet Neurol 2011;10:745-58.

3 Metz I, Radue E-W, Oterino A, et al. Pathology of immune reconstitution inflammatory syndrome in multiple sclerosis with natalizumab-associated progressive multifocal leukoencephalopathy. Acta Neuropath 2012;123:235-45.

4 Gorelik L, Lerner M, Bixler S, et al. Anti-JC virus antibodies: implications for PML risk stratification. Ann Neurol 2010;68:295-303.

5 Ryschkewitsch CF, Jensen PN, Monaco MC, et al. JC virus persistence following progressive multifocal leukoencephalopathy in multiple sclerosis patients treated with natalizumab. Ann Neurol 2010:68:384-91. 